\title{
Resting-State Brain Activity for Early Prediction Outcome in Postanoxic Patients in a Coma with Indeterminate Clinical Prognosis
}

\author{
(DD. Pugin, (D). Hofmeister, (D). Gasche, (DS. Vulliemoz, (DK.-O. Lövblad, (DD. Van De Ville, and (D). Haller
}

\begin{abstract}
BACKGROUND AND PURPOSE: Early outcome prediction of postanoxic patients in a coma after cardiac arrest proves challenging. Current prognostication relies on multimodal testing, using clinical examination, electrophysiologic testing, biomarkers, and structural MR imaging. While this multimodal prognostication is accurate for predicting poor outcome (ie, death), it is not sensitive enough to identify good outcome (ie, consciousness recovery), thus leaving many patients with indeterminate prognosis. We specifically assessed whether resting-state fMRI provides prognostic information, notably in postanoxic patients in a coma with indeterminate prognosis early after cardiac arrest, specifically for good outcome.
\end{abstract}

MATERIALS AND METHODS: We used resting-state fMRI in a prospective study to compare whole-brain functional connectivity between patients with good and poor outcomes, implementing support vector machine learning. Then, we automatically predicted coma outcome using resting-state fMRI and also compared the prediction based on resting-state fMRI with the outcome prediction based on DWI.

RESULTS: Of 17 eligible patients who completed the study procedure (among 351 patients screened), 9 regained consciousness and 8 remained comatose. We found higher functional connectivity in patients recovering consciousness, with greater changes occurring within and between the occipitoparietal and temporofrontal regions. Coma outcome prognostication based on resting-state fMRI machine learning was very accurate, notably for identifying patients with good outcome (accuracy, 94.4\%; area under the receiver operating curve, 0.94). Outcome predictors using resting-state fMRI performed significantly better $(P<.05)$ than DWI (accuracy, $60.0 \%$; area under the receiver operating curve, 0.63 ).

CONCLUSIONS: Indeterminate prognosis might lead to major clinical uncertainty and significant variations in life-sustaining treatments. Resting-state fMRI might bridge the gap left in early prognostication of postanoxic patients in a coma by identifying those with both good and poor outcomes.

ABBREVIATIONS: $\mathrm{CA}=$ cardiac arrest; EEG = electroencephalography; $\mathrm{FC}=$ functional connectivity; ICU = intensive care unit; LOOCV = leave-one-out cross-validation; NPV = negative predictive value; $P P V$ = positive predictive value; rs-fMRI = resting-state fMRI

C ardiac arrest (CA) is an important cause of death in the United States and Europe, with an annual incidence of 110/ $100,000 .^{1,2}$ Only $7.6 \%$ of patients treated for out-of-hospital cardiac arrest survive to hospital discharge. In Europe, 128,000-275,000 individuals per year are treated for out-of-hospital cardiac arrest, and $10 \%$ survive. $^{2,3}$ Due to improvements made in prehospital and intrahospital CA management, a growing number of patients survive the first days following CA, yet can remain unconscious. The

Received August 13, 2019; accepted after revision March 21, 2020.

From the Departments of Intensive Care (D.P., Y.G.), Radiology (J.H.), Neurology (S.V.), and Neuroradiology (K.-O.L.), Geneva University Hospitals, Geneva, Switzerland; Geneva Neuroscience Center (J.H., D.V.D.V.); Radiology and Medical Informatics (J.H., D.V.D.V.); and Faculty of Medicine (S.H.), University of Geneva, Geneva, Switzerland; Institute of Bioengineering (D.V.D.V.), Ecole Polytechnique Fédérale de Lausanne (EPFL), Lausanne, Switzerland; Centre d'Imagerie Rive Droite (S.H.), Geneva, Switzerland; and Department of Surgical Sciences, Radiology (S.H.), Uppsala University, Uppsala, Sweden. main factor determining death in patients with out-of-hospital cardiac arrest admitted to the intensive care unit (ICU), accounting for two-thirds of deaths, is postanoxic brain injury, which leads to a withdrawal of care. Prognostication of patients in a coma can be performed soon after CA (approximately 3 days) and relies on

D. Pugin is funded by a research fellowship of the Faculty of Medicine of Geneva University and by a Templin grant. J. Hofmeister is supported by a Research and Development fund by Geneva University Hospitals. S. Vulliemoz is funded by Swiss National Science Foundation grants 169198 and CRSII5_170873, and K.O. Lövblad, by a Swiss National Science Foundation grant 32003B_160222/1.

Please address correspondence to Deborah Pugin, MD, Neurolntensive Care Unit, Department of Intensive Care Medicine, Geneva University Hospitals, rue Gabrielle Perret Gentil 4, CH-1205 Geneva, Switzerland; e-mail: Deborah.pugin@hcuge.ch

- Indicates open access to non-subscribers at www.ajnr.org

$\equiv$ Indicates article with supplemental on-line appendix and table.

http://dx.doi.org/10.3174/ajnr.A6572 
multimodal testing, using clinical examination, electrophysiologic testing, structural and diffusion neuroimaging, and biomarkers. ${ }^{4-6}$ While this multimodal prognostication is accurate for predicting poor outcome (ie, death), it is not sensitive enough to identify patients with good outcome (ie, consciousness recovery). Thus, even after current multimodal testing, a subset of patients is left with indeterminate prognosis (ie, all patients not identified as having poor outcome) ${ }^{7}$

Several neuroimaging studies have shown that changes on DWI within 5 days after CA predicted poor outcome. ${ }^{8-15}$ Yet the timing of DWI analysis is crucial because diffusion values vary soon after anoxia. ${ }^{10}$ Moreover, while DWI is a strong predictor of poor outcome, it is not sensitive enough to identify patients with good outcomes.

The spontaneous activity of the brain is not random, but rather organized in functional networks. ${ }^{16}$ Resting-state fMRI (rs-fMRI) is a powerful tool for mapping the brain functional connectivity (FC) of patients and healthy volunteers. ${ }^{17}$ Several studies have reported that rs-fMRI could differentiate between states of consciousness in patients with chronic brain damage, with decreased FC correlating with the degree of consciousness impairment. ${ }^{18}$ It has been recently shown that fMRI could detect early signs of consciousness in response to passive stimulation in comatose patients following traumatic brain injury ${ }^{19}$ and that FC strength correlated with favorable long-term outcome in postanoxic patients in a coma. ${ }^{20}$ However, rs-fMRI has yet to be systematically assessed for the early prognostication of the postanoxic patient in a coma.

Our study aimed to predict coma outcome (ie, consciousness recovery versus remaining comatose; namely good-versus-poor outcome) using rs-fMRI and machine learning methods. We focused on cases of particular clinical interest, notably early postanoxic patients in a coma and patients left with indeterminate prognosis after standard multimodal testing.

\section{MATERIALS AND METHODS}

\section{Subjects and Protocol}

The study took place at the Geneva University Hospitals, approved by the local ethics committee for research on human subjects and performed in accordance with the Declaration of Helsinki. We screened all patients comatose following CA admitted to the Geneva University Hospitals' ICU during 4 years ending September 2016.

On admittance, patients comatose following CA were assessed by the ICU team. Those fulfilling the inclusion criteria (see below) and with legal representatives to provide written informed consent were scheduled for early MR imaging (intended to be performed 4 days after CA). All patients still comatose 3 days after CA were considered for inclusion. Patients in a coma were defined as those who did not respond to commands, with Glasgow Coma Scale scores of $\leq 8$ not related to sedation. Patients identified with poor outcome after standard multimodal testing according to the American Academy of Neurology guidelines (generalized myoclonic jerks and bilateral N20 wave abolition or flat electroencephalography [EEG]) were excluded. ${ }^{4,21,22}$ Patients with MR imaging contraindications like pacemakers or extracorporeal membrane oxygenation were excluded. Moreover, major sedation is required for cardiogenic shock treated with extracorporeal membrane oxygenation. This sedation then prevents reliable neuroprognostication in patients. Patients who were conscious and receptive to commands before day 3 or with a history of brain injury were also excluded. Thus, we included only patients whose outcome was left uncertain after current standard multimodal testing. The standard multimodal testing included clinical examination, and particularly pupillary and corneal reflexes, electrophysiologic testing including EEG and somatosensory-evoked potentials, and neuroimaging (mainly MR imaging). ${ }^{21,22}$ Poor outcome was defined as Glasgow Pittsburgh Cerebral Performance Category scale of 4-5 (persistent vegetative state or death). Patients with a good outcome were patients with Glasgow Pittsburgh Cerebral Performance Category of 1-3 (absent, mild, moderate, or severe neurologic disabilities). ${ }^{6,21}$ All patients were treated with normothermia $\left(36^{\circ}\right)$ for the first 24 hours.

MRIs were performed at the radiology department of the Geneva University Hospitals, with patients positioned in the scanner while still intubated. The whole MR imaging scanning session was supervised by a neurointensivist from the ICU team (D.P.) involved in the study; all fMRI sessions were recorded without sedation. If the patient was uncomfortable or moving during imaging, propofol was added after fMRI sequences were recorded. Surviving patients were followed up at 3,6, and 12 months with the mRS, Glasgow Outcome Scale, Cerebral Performance Category scale, and Disability Rating Scale. Information was gathered by physicians blinded to the fMRI results. This process was undertaken either by the physician in charge of inpatients or by phone interview for patients who were discharged.

\section{MR Imaging Acquisition and Analysis}

All patients included underwent MR imaging with a protocol including a 3D-T1 image, DWI, and a blood oxygen level-dependent resting-state sequence (On-line Appendix).

DWI derived from DTI with 30 directions plus the $\mathrm{B}_{0}$ of each patient were analyzed to predict coma outcome, as per the description of Wijman et al, ${ }^{9}$ by computing the percentage of brain volume with an ADC value of $<650 \times 10^{-6} \mathrm{~mm}^{2} / \mathrm{s}$. Patients with $>10 \%$ brain volume with abnormal $\mathrm{ADC}$ values were assigned to the poor outcome group, while the others were assigned to the good outcome group, as proposed by Wijman et al. fMRI and 3D-T1 images were preprocessed for each subject independently, following standard protocol ${ }^{23,24}$ (On-line Appendix), including normalization into Montreal Neurological Institute space, to assess whole-brain resting-state activity of each comatose patient and to compare the brain network topology of patients with good and poor outcomes. Thus, we statistically compared brain-network activity derived from fMRI between groups with good and poor outcomes, following standard procedure $^{25}$ (On-line Appendix).

\section{Coma Outcome Prediction}

We ultimately aimed to base predictions of coma outcome on resting-brain activity measured with $\mathrm{PMRI}$. Thus, we trained a machine learning classifier to identify patients with good and poor outcomes based on brain network topology derived from fMRI (On-line Appendix). Classification performance was assessed using leaveone-out cross-validation (LOOCV) methods, because our sample size was relatively small, allowing computing prediction-accuracy measures (overall accuracy, sensitivity, specificity, positive prediction value $[\mathrm{PPV}]$, and negative predictive value $[\mathrm{NPV}])$. Finally, we 
statistically compared the prediction accuracy based on restingbrain activity with that using DWI (On-line Appendix).

\section{RESULTS}

\section{Demographic Data}

We screened 351 patients comatose following CA, 17 of whom underwent the whole protocol and were included in the final analysis (Fig 1). At admission, 7/17 patients had areactive mydriasis, 1 had reactive mydriasis, and 9 had a reactive miosis. At day 3 , three patients had reactive mydriasis and 14 had reactive miosis. All patients had controlled normothermia $\left(36^{\circ}\right)$ for the first 24 hours. Of these 17, nine regained consciousness (mean, $4 \pm$ 7.8 days after the MR imaging session; good outcome group); the remaining 8 did not and finally died (poor outcome group). Both groups were similar in terms of demographic data, apart from the time until the return of spontaneous circulation, which was $50 \%$ longer in the poor outcome group (Table 1). Both groups were scanned in the same time window after hospital admission, when

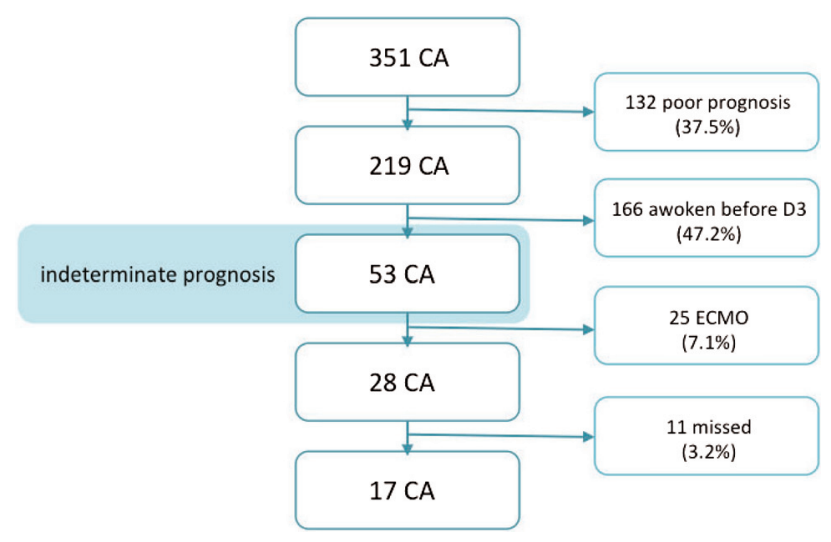

FIG 1. Inclusion flow chart. D indicates day; ECMO, extracorporeal membrane oxygenation. no survival prognosis could be made on the basis of their clinical status or standard multimodal testing (Table 2). No sedation was used during fMRI. If the patient was uncomfortable or moving during imaging, propofol was added after fMRI sequences were recorded. The mean delay between propofol cessation and MR imaging was 41 hours (minimum, 24 hours; maximum, 120 hours) and 82 hours for midazolam (minimum, 19 hours; maximum, 264 hours).

At 1 year, 5 patients with good outcome had Disability Rating Scale scores of $\leq 1$, eight had Glasgow Outcome Scale scores of $\geq 4$ and a Cerebral Performance Category score of $\leq 2$, and 7 had $\mathrm{mRS} \leq 1$ (ie, they were almost completely independent). Two patients remained partially or totally dependent, essentially due to cognitive disorders. Of the patients who died, 1 patient had a second CA, 5 remained in a persistent comatose state leading to care withdrawal, and 2 had adverse outcomes following extubation.

\section{Network Topology}

We initially compared network topology between the good and poor outcome groups at each individual functional connection (ie, edge weight). We first visually inspected the mean wholebrain network of each group, observing much better-preserved network architecture in patients with good outcomes than in those with poor outcomes. We then statistically compared network topology between groups (Fig 2). We observed a significant increase in connectivity between many brain regions in the good outcome group (Fig 3). The strongest and most extensive increased connectivity in the good outcome group (versus poor outcome) occurred within and between the occipital, parietal, temporal, and frontal regions. We observed significant changes within the occipital nodes and between the fronto-occipital, temporo-occipital, and occipitolimbic nodes. We also observed increased connectivity within the parietal nodes and among the parietofrontal, parietocentral, and parietotemporal nodes. The temporal lobe showed increased connectivity within the temporomedial nodes and among the temporofrontal, temporocentral,

Table 1: Demographic and clinical characteristics of patients

\begin{tabular}{|c|c|c|c|c|}
\hline & All $(n=17)$ & Good Outcome $(n=9)$ & Poor Outcome $(n=8)$ & $P$ Value \\
\hline Male & $14(82.4 \%)$ & $7(77.8 \%)$ & $7(87.5 \%)$ & 6 \\
\hline Age (yr) & $57.5 \pm 15.6$ & $52.2 \pm 13.2$ & $63.4 \pm 16.9$ & .07 \\
\hline CA witnessed & 17 (100\%) & $9(100 \%)$ & $8(100 \%)$ & NA \\
\hline CA outside hospital & $13(76.5 \%)$ & $8(88.9 \%)$ & $5(62.5 \%)$ & .2 \\
\hline Time to ROSC (min) & $23.4 \pm 15.9$ & $17 \pm 10.2$ & $30.62 \pm 18.6$ & .03 \\
\hline \multicolumn{5}{|l|}{ First monitored rhythm } \\
\hline VF/VT & $10(58.6 \%)$ & 7 (77.8\%) & $3(37.5 \%)$ & \\
\hline PEA & $2(11.7 \%)$ & $1(11.1 \%)$ & $1(12.5 \%)$ & \\
\hline Asystole & $5(29.3 \%)$ & $1(11.1 \%)$ & $4(50 \%)$ & \\
\hline Hospital stay (days) & $19 \pm 20$ & $28 \pm 23.9$ & $9 d \pm 6.2$ & .97 \\
\hline ICU stay (days) & $9 \pm 5.4$ & $10 \pm 5.1$ & $8 \pm 6.1$ & .71 \\
\hline ETT length (days) & $7 \pm 4.3$ & $8 \pm 4.9$ & $7 \pm 3.8$ & .65 \\
\hline Time before fMRI (days) & $4 \pm 2.9$ & $5 \pm 3.4$ & $4 \pm 2.2$ & .72 \\
\hline Myoclonia & 2 (11.8\%) & $1(11.1 \%)$ & $1(12.5 \%)$ & .92 \\
\hline Lactate (mmol/L) & $7.2 \pm 4.1$ & $7.1 \pm 3.7$ & $7.36 \pm 4.8$ & .43 \\
\hline \multicolumn{5}{|l|}{ First GCS } \\
\hline 3 & $14(82.4 \%)$ & $7(77.8 \%)$ & $6(87.5 \%)$ & \\
\hline 6 & $1(5.9 \%)$ & $1(11.1 \%)$ & & \\
\hline 7 & 2 (11.7\%) & $1(11.1 \%)$ & $1(12.5 \%)$ & \\
\hline
\end{tabular}

Note:-ROSC indicates return of spontaneous circulation; VF/VT, ventricular fibrillation/tachycardia; PEA, pulseless electrical activity; ETT, endotracheal intubation; GCS, Glasgow Coma Scale; NA, not applicable. 
temporoparietal, and temporo-occipital nodes. Finally, we observed significant increased connectivity among the frontoparietal, frontocentral, frontotemporal, and fronto-occipital nodes.

We also observed a less significant increase in focal connectivity in the poor outcome group (versus good outcome). The poor outcome group exhibited higher focal activity within the temporosuperior and temporopolar regions, as well as between these temporosuperior and temporopolar regions and occipital areas.

Taken together, these results suggest that patients who eventually recover consciousness (ie, the good outcome group) initially exhibit higher activity within the occipital areas and between these areas and widely distributed cortical regions, except for the temporosuperior and temporopolar regions. Conversely, patients who ultimately die (ie, poor outcome) initially show higher activity focally, among the occipital, temporosuperior, and temporopolar regions, as well as within these areas.

\section{Coma Outcome Prediction}

Diffusion Imaging. The percentage of brain volume with ADC values of $<650 \times 10^{-6} \mathrm{~mm}^{2} / \mathrm{s}$ was used to predict coma outcome, with a threshold set at $10 \%$ as proposed by Wijman et al. ${ }^{9}$ The model achieved $64.7 \%$ accuracy in discriminating good and poor coma outcomes (poor outcome prediction sensitivity, $25.0 \%$; specificity, $100.0 \%$; PPV, 100\%; NPV 60.0\%; area under the receiver operating characteristic curve, 0.625).

Resting-State fMRI. We then used rsfMRI to train a machine learning ground reactivity or only stimulus-induced discharges; LPDs, lateralized periodic discharges; RDA. rhythmic $\Delta$ activity.

\section{Table 2: EEG pattern}

\begin{tabular}{lll}
\hline Malignant EEG pattern & $0 / 9$ & $0 / 8$ \\
Periodic features & $5 / 9$ none, 4/9 RDA & $5 / 8$ none; 2/8 LPDs, 1/8 RDA \\
Unreactive EEG & $2 / 9$ unreactive, 7/9 reactive & $6 / 8$ unreactive, 2/8 reactive \\
Seizure & $0 / 9$ & $0 / 8$ \\
Disorganized background & $1 / 9$ & $4 / 8$ \\
\hline
\end{tabular}

Note:-Malignant EEG pattern indicates suppressed background with or without continuous periodic discharges, burst-suppression, abundant periodic discharges, or rhythmic epileptiform transients, electrographic seizure, discontinuous or low-voltage background, reversed anterior-posterior gradient; Unreactive EEG, absence of back-

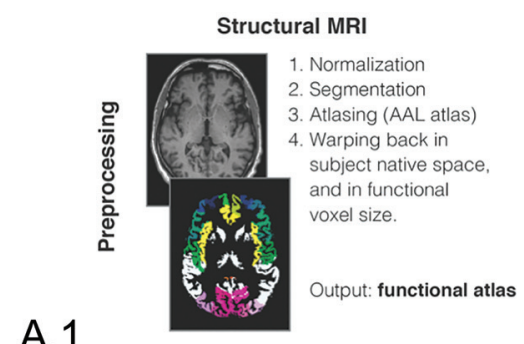

A.1

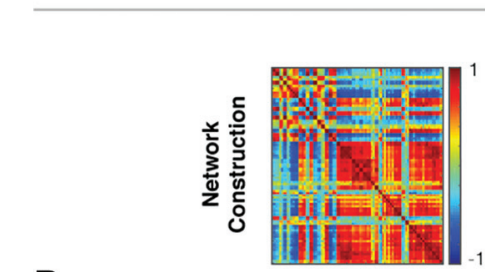

B

\section{Functional MRI}

1. Realignment

2. Smoothing $(8 \mathrm{~mm})$

3. Coregistration with $\mathrm{T} 1$

4. Voxel timecourse extraction

5. Noise removal and detrending

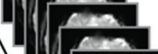

Output: voxel timecourses
A.2

1. Averaging of fMRI voxels timecourses' for each brain region

of the functional atlas.

Pearson correlation computation, between the averaged

time course of each pair of brain region $(\mathrm{N}=90)$ of the

functional atlas.

3. Fisher r-to-z transformation.

Output: one NxN connectivity matrix (CM) per patient

(i.e. whole brain connectivity matrix)

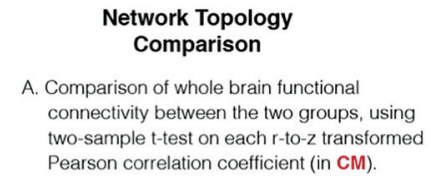

C.1

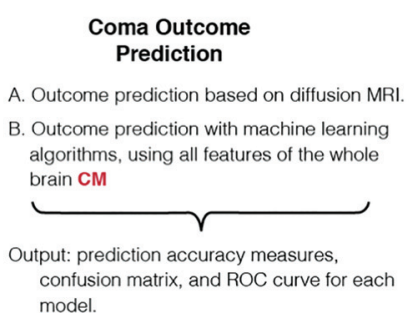

Coma Outcome

A. Outcome prediction based on diffusion MRI.

B. Outcome prediction with machine learning algorithms, using all features of the whole brain $\mathrm{CM}$

Output: prediction accuracy measures,

C.2 model

FIG 2. Analysis pipeline. Structural (A.I) and functional (A.2) MRIs of each patient are first preprocessed to extract time courses of each brain voxel. $B$, An averaged time course is then computed for each of 90 brain regions of the Automated Anatomical Labeling atlas, and recursive Pearson correlations are computed between each brain region pair, to obtain a whole-brain connectivity network for each patient. C.1, Networks of patients with good and poor outcomes are compared using a 2-sample $t$ test corrected for multiple comparisons. C.2, A support vector machine classifier is also trained on the connectivity networks of each patient to discriminate patients with good and poor outcomes. $\mathrm{N}$ indicates brain regions; $\mathrm{CM}$, connectivity matrix; ROC, receiver operating characteristic; AAL, Automated Anatomical Labeling. 


\section{Good outcome}

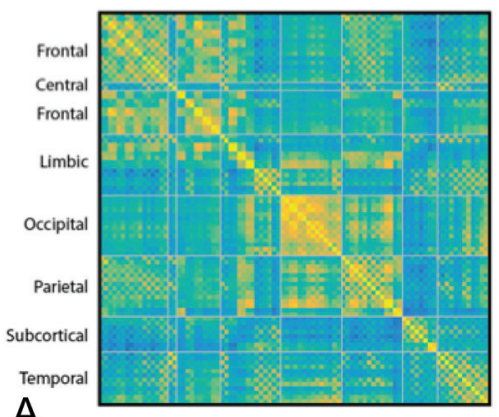

A

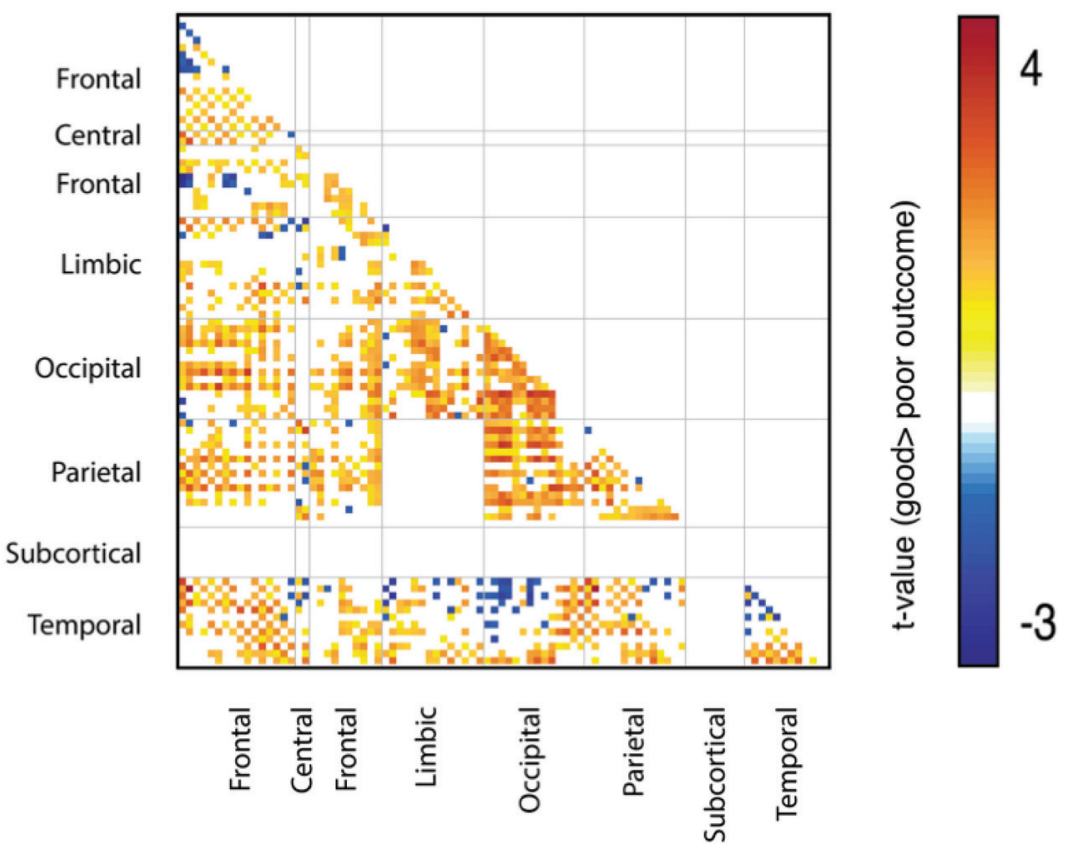

FIG 3. Statistical analysis of the functional network topology of good and poor outcome groups. $A$, Mean functional connectivity matrices of good and poor outcome groups. B, Statistical comparison of functional connectivity among all 90 brain regions of the Automated Anatomical Labeling atlas, with a $t$-score given only for significant comparisons $(P<.05$ after correction for multiple comparisons).

algorithm in automatic prediction of coma outcome. We used all the features derived from connectivity matrices of all patients (4005 features $\times 17$ subject matrices) to predict coma outcome using a machine learning classifier (see above) and LOOCV, with and without dimension reduction (using principal component analysis). Without dimension reduction, our linear support vector machine classifier achieved $66.7 \%$ overall prediction accuracy. With dimension reduction applied during LOOCV, we achieved 94.4\% overall prediction accuracy using a linear support vector machine classifier $(C=0.1)$. This model achieved $100 \%$ sensitivity in predicting poor outcome, $87.5 \%$ specificity, $100 \%$ PPV, and $90 \%$ NPV, for an area under the curve of 0.938 . Adding a DWI feature (ie, the percentage of brain volume with abnormal ADC values) to the classification model did not change the prediction accuracy.

Finally, we compared automatic outcome prediction based on rs-fMRI and machine learning with prediction based on DWI following the method proposed by Wijman et al. ${ }^{9}$ We found that the machine learning classifier trained on rs-fMRI features yielded significantly better prediction accuracy than the DWI method $(P=.025$, two-sided; Fig 4$)$.

\section{DISCUSSION}

We wanted to take advantage of the advances in machine learning to assess how functional neuroimaging can improve early prognostication of postanoxic patients in a coma left with an indeterminate prognosis after standard multimodal testing. To this end, we trained and assessed the performance of a machine learning classifier in discriminating between patients with good and poor outcomes based on FC patterns derived from rs-fMRI. We found that automatic prediction based on functional neuroimaging coupled with machine learning methods yielded better prognostication compared with current diffusion neuroimaging methods, especially in terms of identifying patients who would subsequently recover consciousness (ie, good outcome). Additionally, we report on the early changes observed in cortico-cortical FC between good and poor outcome groups in a very difficult group of patients with indeterminate outcome following standard clinical and electrophysiologic tests.

\section{Whole-Brain Functional Connectivity Predicts Coma Outcome}

Postanoxic patients in a coma very often undergo MR imaging to identify potential brain lesions and determine neurologic outcome. Conventional MR imaging based solely on structural images was found to underestimate the severity of lesions, notably when performed soon after CA. ${ }^{26}$ More recently, DWI was proposed to overcome these failings. It proved sensitive in detecting ischemic lesions soon after CA (2-5 days). ${ }^{27}$ It was thus proposed as a neuroimaging tool for early prognostication of postanoxic patients in a coma, because the extent of lesions observed on DWI correlates with neurologic outcome. ${ }^{9,26,28-30}$ Recent studies have reported that quantitative DWI based on the extent of impaired brain volume (with a threshold set at $10 \%$, as in our study) offers high specificity for predicting poor outcome in postanoxic patients in a coma, with PPVs between $96 \%{ }^{29}$ and $100 \% .{ }^{9}$ However, it was less sensitive in identifying patients with good outcome, with NPVs for poor outcome of between $54 \%{ }^{31}$ and $73 \%$. $^{9}$ Similarly, in our study, we found that quantitative DWI offered very high specificity for predicting poor outcome (100\% PPV), yet lower 

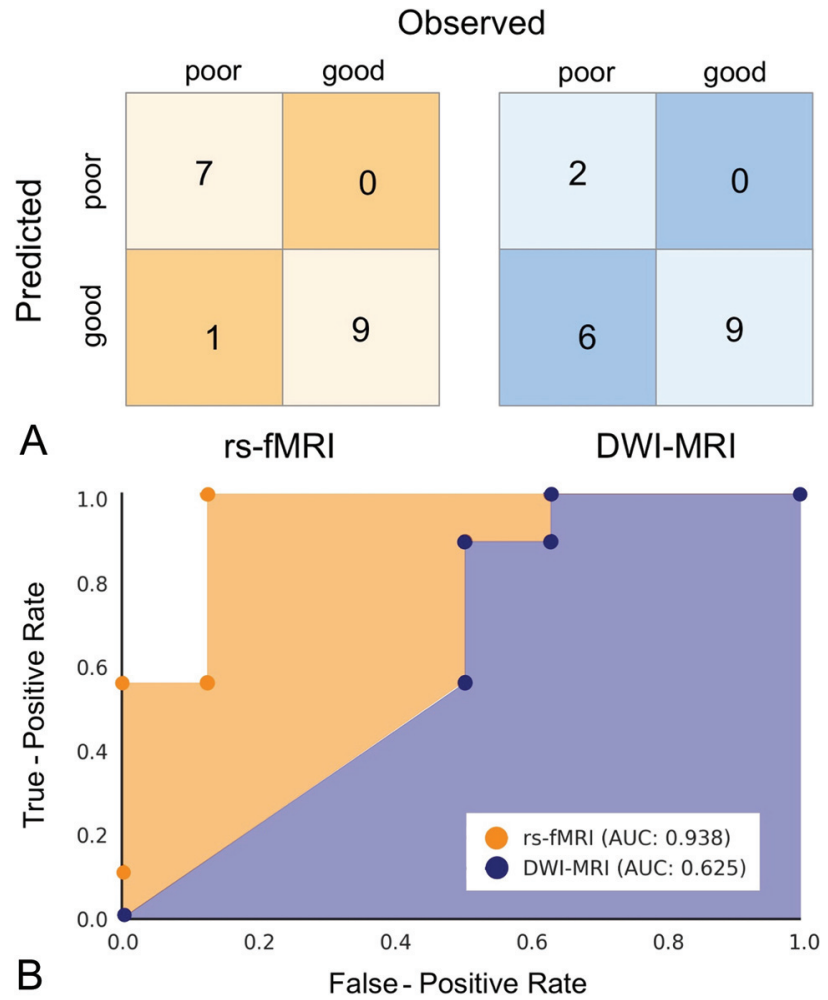

FIG 4. Outcome classification using rs-fMRI and DWI. A, Confusion matrix for the rs-fMRI classification using support vector machine (left, in orange) and DWI classifications using the model proposed by Wijman et al, 2009 (right, in blue). B, Receiver operating characteristic curves for rs-fMRI and DWI classification models. AUC indicates area under the curve.

performance for predicting good outcome (60.0\% NPV). Thus, both our results and those of recent studies demonstrate that all patients not identified as having poor outcome retain indeterminate prognosis following quantitative DWI analysis. These findings might lead to substantial clinical uncertainty and significant variations in life-sustaining treatments. ${ }^{7}$

Rs-fMRI is available in most specialized large hospitals and has been used successfully to evaluate the state of consciousness of patients with chronic disorders of consciousness or traumatic brain injuries. ${ }^{18}$ Here, we used rs-fMRI for early prediction of postanoxic coma outcome in patients left with indeterminate prognosis after standard multimodal testing. We accurately predicted the outcome of $16 / 17$ (94.1\%) patients in a coma based on their whole-brain FC at rest. Using a support vector machine classifier with LOOCV was found to offer very high accuracy (100\% PPV) for identifying patients with poor outcome, similar to quantitative DWI. However, much higher accuracy for predicting consciousness recovery (ie, NPV for predicting poor outcome) was achievable using rs-fMRI (90\%) compared with quantitative DWI (60.0\%). This outcome is particularly interesting because there is a critical need to test for predicting good outcome but no widespread and wellaccepted tool.

A recent study by Sair et $\mathrm{al}^{20}$ also evaluated postanoxic patients in a coma, finding that higher default mode network connectivity assessed soon after CA using rs-fMRI correlates with favorable outcome at 1 year, compared with patients with unfavorable outcome. Although the author investigated the long-term neurologic outcome, these results are in line with ours because they also demonstrate that whole-brain FC correlates with outcome in postanoxic patients in a coma. Here, we provide further evidence that rs-fMRI could be used in patients comatose soon after CA. The mean time between CA and fMRI in our cohort was $4 \pm 2.9$ days versus $12.6 \pm 5.6$ days for Sair et al. All our patients underwent fMRI between 2 and 8 days after CA, except one who underwent fMRI 13 days after CA due to prior treatment with extracorporeal membrane oxygenation. Despite the delay in recording fMRI, this patient's whole-brain FC was extremely similar to that of the others in the good outcome group. Moreover, our study specifically focused on a group of patients left with indeterminate prognosis after current multimodal testing, excluding patients with catastrophic outcome or patients already conscious, and revealed that automatic outcome prognostication using machine learning based on rs-fMRI outperforms current state-of-the-art DWI methods. Finally, the study by Sair et al did not test whole-brain FC as in the current study. Rather it tested within and between standard topographic network connectivity in terms of the relationship to the outcome.

Overall, our results demonstrate that rs-fMRI achieves significantly better prognostication of postanoxic patients in a coma left with an indeterminate prognosis after standard multimodal testing than DWI, notably with very high accuracy and higher specificity in predicting good outcome. This finding could have a great impact on the identification and clinical management of patients with good outcome, reducing the uncertainty that remains after current multimodal prognostication.

\section{Higher Whole-Brain Functional Connectivity in Patients Recovering from Coma}

Whole-brain activity varies greatly depending on states of consciousness, both in healthy volunteers (eg, during sleep or sedation $)^{31,32}$ and in patients with disorders of consciousness. ${ }^{33}$ Most neuroimaging studies investigating disorders of consciousness have focused on patients with chronic impaired consciousness or compared them with healthy volunteers. ${ }^{18,34,35}$ Here, we investigated changes in brain activity in postanoxic patients comatose shortly after CA and found differences in whole-brain FC observable early on between patients with good and poor outcomes, even in the absence of extended lesions on morphologic MR imaging.

Patients with good-versus-poor outcome exhibited higher FC strength between widely distributed cortical areas, especially in the occipital, frontal, parietal, and inferior temporal cortices. This suggests that patients who subsequently recover from comas have better preserved cortico-cortical connectivity, similar to the higher cortico-cortical connectivity in higher states of consciousness observed in patients with chronic disorders of consciousness ${ }^{18,36}$ and healthy volunteers under sedation. ${ }^{37,38}$ In our study, we could not identify significant connections between the cortex and subcortical regions despite the known role of thalamocortical and other cortical-subcortical connections in consciousness. We reason that this issue is because those connections tend to be weak in states of reduced consciousness, and the patients in our studies were indeed unconscious. 
Our results also suggest that recovery from coma relies on better preserved whole-brain cortico-cortical connectivity, plausibly between the frontal and parietal areas, as shown by Crone et $\mathrm{al}^{39}$ in patients with chronic disorders of consciousness. In our study, the most extensive changes were observed in visual areas (ie, occipital and inferior temporal regions), with higher brain FC exhibited by patients with good outcome, even if they kept their eyes closed in the scanner. Our results are thus in line with the finding of an increased connectivity of visual areas with increased levels of consciousness when comparing chronic comatose patients and healthy volunteers. ${ }^{40}$ This is also congruent with the preserved ability for sensory processing ${ }^{41}$ and mental imaging ${ }^{42}$ observed in patients with a minimal conscious state compared with a vegetative state. Studies assessing brain dynamic FC activity could bring further insight into how the functional brain networks of these patients are reorganized.

Patients with poor outcomes had few higher FC strengths among the focal cortical areas, notably in the temporopolar regions. Given the close link between the temporopolar regions and other limbic areas, ${ }^{43}$ these findings might relate to limbic hyperconnectivity observed in patients in chronic comatose and vegetative states. $^{44}$

Overall, we found much higher cortico-cortical connectivity in patients with good outcome, consistent with the findings of higher cortico-cortical connectivity with higher levels of consciousness in both patients and healthy volunteers in other studies. Most interesting, the changes observed in acute postanoxic patients in a coma resemble those reported in patients with chronic consciousness disorders, notably concerning the global higher whole-brain FC found in patients who subsequently recover consciousness. ${ }^{35}$ This finding suggests that the changes in whole-brain connectivity observed in chronic disorders of consciousness may develop soon after loss of consciousness. Future studies should, however, repeatedly investigate comatose patients to find further evidence supporting whole-brain FC changes persisting from acute-tochronic disorders of consciousness.

In our study, we could not identify significant connections between the cortex and subcortical regions despite the known role of thalamocortical and other cortical-subcortical connections in consciousness. We reason that this issue is because those connections tend to be weak in states of reduced consciousness, and the patients in our study were indeed unconscious.

\section{Limitations}

Although our results appear promising, they need to be further validated in a larger prospective cohort study, notably using independent training and testing samples to prove the accuracy of rsfMRI for early outcome prognostication in postanoxic patients in a coma. A major limitation of our study is the small number of patients included, despite screening 351 consecutive patients and identifying 28/351 (8\%) eligible ones (increasing to 53/351 [15\%] patients if adding the 25 patients with an extracorporeal membrane oxygenation procedure, limiting MR imaging use). We excluded all patients conscious before day 3 or identified with poor prognosis using multimodal testing according to the American Academy of Neurology guidelines. ${ }^{4}$ Moreover, we included only patients who were sedation-free during the fMRI session, to avoid drug-induced changes in brain activity. Concerning DWI, previous studies specifically assessed the impact of timing on DWI accuracy. This has not yet been done with fMRI. The essential point of the current study was to perform fMRI early after cardiac arrest and to focus on those cases with uncertain outcome because this scenario is, in our opinion, the most challenging yet, at the same time, clinically most relevant one. The effect of timing of fMRI assessment with respect to coma onset should be specifically assessed, yet this is beyond the scope of our current article. The current investigation did not include a healthy control group because the objective of the current study was to discriminate patients with good-versuspoor outcomes. An additional control group is not necessary for this objective. Although scientifically interesting, the discrimination of patients versus controls is irrelevant from a clinical perspective. Our strict selection of only patients left with indeterminate prognosis after standard multimodal testing soon after CA represents a population in which such diagnostic tests would be crucial for clinical decision-making. On the basis of our inclusion rate, we anticipate that rs-fMRI-based prognostication could be valuable for around $8 \%-15 \%$ of patients in a postanoxic comatose state.

\section{CONCLUSIONS}

Using machine learning classification methods, we found that rsfMRI yields a valuable contribution in the prognostication of postanoxic patients in a coma left with an indeterminate prognosis after standard multimodal testing, notably offering high accuracy for identifying patients with both good (ie, patients who will regain consciousness) and poor (ie, patients who will evolve poorly) outcomes. Our results might thus bridge the gap left in early prognostication of postanoxic patients in a coma by achieving significantly better outcome prediction than current DWI methods. Moreover, our study may contribute to improving understanding of brain FC changes occurring after loss of consciousness, with the early changes reported here mirroring changes observed in chronic comatose patients.

\section{ACKNOWLEDGMENTS}

The authors thank Dr Gabrielle Cremer from Cremer Consulting SARL for expert English editing.

Disclosures: Sven Haller-UNRELATED: Payment for Lectures Including Service on Speakers Bureaus: GE Healthcare. Serge Vulliemoz-RELATED: Grant: Swiss National Research Foundation, Comments: Principal Investigator of 1 current grant and coPrincipal Investigator of another*; Support for Travel to Meetings for the Study or Other Purposes: Swiss National Research Foundation, Comments: travel funding in the context of the grant*; UNRELATED: Stock/Stock Options: Epilog, Belgium, Comments: shareholder of Epilog, a start-up providing electroencephalogram analysis for clinical and research groups. *Money paid to the institution.

\section{REFERENCES}

1. Mozaffarian D, Benjamin EJ, Go AS, et al; American Heart Association Statistics Committee and Stroke Statistics Subcommittee. Heart disease and stroke statistics-2015 update: a report from the American Heart Association. Circulation 2015;131:e29-322 CrossRef Medline 
2. Atwood C, Eisenberg MS, Herlitz J, et al. Incidence of EMS-treated out-of-hospital cardiac arrest in Europe. Resuscitation 2005;67:7580 CrossRef Medline

3. Grasner JT, Lefering R, Koster RW, et al. EuReCa ONE-27 Nations, ONE Europe, ONE Registry: a prospective one-month analysis of out-of-hospital cardiac arrest outcomes in 27 countries in Europe. Resuscitation 2016;105:188-95 CrossRef Medline

4. Wijdicks EF, Hijdra A, Young GB, et al; Quality Standards Subcommittee of the American Academy of Neurology. Practice parameter: prediction of outcome in comatose survivors after cardiopulmonary resuscitation (an evidence-based review)-report of the Quality Standards Subcommittee of the American Academy of Neurology. Neurology 2006;67:203-10 CrossRef Medline

5. Sandroni C, Cavallaro F, Callaway CW, et al. Predictors of poor neurological outcome in adult comatose survivors of cardiac arrest: a systematic review and meta-analysis, Part 2: patients treated with therapeutic hypothermia. Resuscitation 2013;84:1324-38 CrossRef Medline

6. Sandroni C, Cavallaro F, Callaway CW, et al. Predictors of poor neurological outcome in adult comatose survivors of cardiac arrest: a systematic review and meta-analysis, Part 1: patients not treated with therapeutic hypothermia. Resuscitation 2013;84:1310-23 CrossRef Medline

7. Weijer C, Bruni T, Gofton T, et al. Ethical considerations in functional magnetic resonance imaging research in acutely comatose patients. Brain 2016;139:292-99 CrossRef Medline

8. Mlynash M, Campbell DM, Leproust EM, et al. Temporal and spatial profile of brain diffusion-weighted MRI after cardiac arrest. Stroke 2010;41:1665-72 CrossRef Medline

9. Wijman CA, Mlynash M, Caulfield AF, et al. Prognostic value of brain diffusion-weighted imaging after cardiac arrest. Ann Neurol 2009;65:394-402 CrossRef Medline

10. Choi SP, Park KN, Park HK, et al. Diffusion-weighted magnetic resonance imaging for predicting the clinical outcome of comatose survivors after cardiac arrest: a cohort study. Crit Care 2010;14:R17 CrossRef Medline

11. Rossetti AO, Oddo M, Logroscino G, et al. Prognostication after cardiac arrest and hypothermia: a prospective study. Ann Neurol 2010;67:301-07 CrossRef Medline

12. Stevens RD, Hannawi Y, Puybasset L. MRI for coma emergence and recovery. Curr Opin Crit Care 2014;20:168-73 CrossRef Medline

13. Park JS, Lee SW, Kim H, et al. Efficacy of diffusion-weighted magnetic resonance imaging performed before therapeutic hypothermia in predicting clinical outcome in comatose cardiopulmonary arrest survivors. Resuscitation 2015;88:132-37 CrossRef Medline

14. Hirsch KG, Mlynash M, Jansen S, et al. Prognostic value of a qualitative brain MRI scoring system after cardiac arrest. J Neuroimaging 2015;25:430-37 CrossRef Medline

15. Keijzer HM, Hoedemaekers CW, Meijer FJ, et al. Brain imaging in comatose survivors of cardiac arrest: pathophysiological correlates and prognostic properties. Resuscitation 2018;133:124-36 CrossRef Medline

16. Shehzad Z, Kelly AM, Reiss PT, et al. The resting brain: unconstrained yet reliable. Cereb Cortex 2009;19:2209-29 CrossRef Medline

17. Sharp DJ, Scott G, Leech R. Network dysfunction after traumatic brain injury. Nat Rev Neurol 2014;10:156-66 CrossRef Medline

18. Vanhaudenhuyse A, Noirhomme Q, Tshibanda LJ, et al. Default network connectivity reflects the level of consciousness in non-communicative brain-damaged patients. Brain 2010;133:161-71 CrossRef Medline

19. Edlow BL, Chatelle C, Spencer CA, et al. Early detection of consciousness in patients with acute severe traumatic brain injury. Brain 2017;140:2399-2414 CrossRef Medline

20. Sair H, Hannawi Y, Li S, et al; For the Neuroimaging for Coma Emergence and Recovery (NICER) Consortium. Early functio- nal connectome integrity and 1-year recovery in comatose survivors of cardiac arrest. Radiology 2018;287:247-55 CrossRef Medline

21. Callaway CW, Donnino MW, Fink EL, et al. Part 8: Post-Cardiac Arrest Care:-2015 American Heart Association Guidelines Update for Cardiopulmonary Resuscitation and Emergency Cardiovascular Care. Circulation 2015;132:S465-82 CrossRef Medline

22. Nolan JP, Soar J, Cariou A, et al. European Resuscitation Council and European Society of Intensive Care Medicine Guidelines for Postresuscitation Care 2015: Section 5 of the European Resuscitation Council Guidelines for Resuscitation 2015. Resuscitation 2015;95:20222 CrossRef Medline

23. Leonardi N, Richiardi J, Gschwind M, et al. Principal components of functional connectivity: a new approach to study dynamic brain connectivity during rest. Neuroimage 2013;83:937-50 CrossRef Medline

24. Richiardi J, Eryilmaz H, Schwartz S, et al. Decoding brain states from fMRI connectivity graphs. Neuroimage 2011;56:616-26 CrossRef Medline

25. Meskaldji DE, Fischi-Gomez E, Griffa A, et al. Comparing connectomes across subjects and populations at different scales. Neuroimage 2013;80:416-25 CrossRef Medline

26. Wijdicks EF, Campeau NG, Miller GM. MR imaging in comatose survivors of cardiac resuscitation. AJNR Am J Neuroradiol 2001;22:156165 CrossRef Medline

27. Mintorovitch J, Moseley ME, Chileuitt L, et al. Comparison of diffusion- and T2-weighted MRI for the early detection of cerebral ischemia and reperfusion in rats. Magn Reson Med 1991;18:39-50 CrossRef Medline

28. Hastie T, Tibshirani R, Friedman J. The Elements of Statistical Learning: Data Mining, Inference, and Prediction. Springer; 2009

29. Hirsch KG, Mlynash M, Eyngorn I, et al. Multi-center study of diffusion-weighted imaging in coma after cardiac arrest. Neurocrit Care 2016;24:82-89 CrossRef Medline

30. $\mathrm{Wu} \mathrm{X}, \mathrm{Zou} \mathrm{Q}, \mathrm{Hu} \mathrm{J}$, et al. Intrinsic functional connectivity patterns predict consciousness level and recovery outcome in acquired brain injury. J Neurosci 2015;35:12932-46 CrossRef Medline

31. Boveroux P, Vanhaudenhuyse A, Bruno MA, et al. Breakdown of within- and between-network resting state functional magnetic resonance imaging connectivity during propofol-induced loss of consciousness. Anesthesiology 2010;113:1038-53 CrossRef Medline

32. Peltier SJ, Kerssens C, Hamann SB, et al. Functional connectivity changes with concentration of sevoflurane anesthesia. Neuroreport 2005; 16:285-88 CrossRef Medline

33. Laureys S, Schiff ND. Coma and consciousness: paradigms (re) framed by neuroimaging. Neuroimage 2012;61:478-91 CrossRef Medline

34. Owen AM, Coleman MR. Functional neuroimaging of the vegetative state. Nat Rev Neurosci 2008;9:235-43 CrossRef Medline

35. Hannawi $\mathrm{Y}$, Lindquist MA, Caffo BS, et al. Resting brain activity in disorders of consciousness: a systematic review and meta-analysis. Neurology 2015;84:1272-80 CrossRef Medline

36. Boly M, Tshibanda L, Vanhaudenhuyse A, et al. Functional connectivity in the default network during resting state is preserved in a vegetative but not in a brain dead patient. Hum Brain Mapp 2009;30:23932400 CrossRef Medline

37. Greicius MD, Kiviniemi V, Tervonen O, et al. Persistent defaultmode network connectivity during light sedation. Hum Brain Mapp 2008;29:839-47 CrossRef Medline

38. Noirhomme Q, Soddu A, Lehembre R, et al. Brain connectivity in pathological and pharmacological coma. Front Syst Neurosci 2010;4:160 CrossRef Medline 
39. Crone JS, Soddu A, Holler Y, et al. Altered network properties of the fronto-parietal network and the thalamus in impaired consciousness. Neuroimage Clin 2014;4:240-48 CrossRef Medline

40. Achard S, Delon-Martin C, Vertes PE, et al. Hubs of brain functional networks are radically reorganized in comatose patients. Proc Natl Acad Sci USA 2012;109:20608-13 CrossRef Medline

41. Demertzi A, Antonopoulos G, Heine L, et al. Intrinsic functional connectivity differentiates minimally conscious from unresponsive patients. Brain 2015;138:2619-31 CrossRef Medline
42. Monti MM, Vanhaudenhuyse A, Coleman MR, et al. Willful modulation of brain activity in disorders of consciousness. $N$ Engl J Med 2010;362:579-89 CrossRef Medline

43. Chabardes S, Kahane P, Minotti L, et al. Anatomy of the temporal pole region. Epileptic Disord 2002;4(Suppl 1):S9-15 Medline

44. Di Perri C, Bastianello S, Bartsch AJ, et al. Limbic hyperconnectivity in the vegetative state. Neurology 2013;81:1417-24 CrossRef Medline 